\title{
MEAN-VARIANCE HEDGING WITH RANDOM VOLATILITY JUMPS
}

\author{
PAOLO GUASONI AND FRANCESCA BIAGINI
}

\begin{abstract}
We introduce a general framework for stochastic volatility models, with the risky asset dynamics given by:$$
d X_{t}(\omega, \eta)=\mu_{t}(\eta) X_{t}(\omega, \eta) d t+\sigma_{t}(\eta) X_{t}(\omega, \eta) d W_{t}(\omega)
$$

where $(\omega, \eta) \in\left(\Omega \times H, \mathcal{F}^{\Omega} \otimes \mathcal{F}^{H}, P^{\Omega} \otimes P^{H}\right)$.

In particular, we allow for random discontinuities in the volatility $\sigma$ and the drift $\mu$. First we characterize the set of equivalent martingale measures, then compute the mean-variance optimal measure $\tilde{P}$, using some results of Schweizer on the existence of an adjustment process $\beta$.

We show examples where the risk premium $\lambda=\frac{\mu-r}{\sigma}$ follows a discontinuous process, and make explicit calculations for $\tilde{P}$.
\end{abstract}

\section{INTRODUCTION}

The introduction of the mean-variance approach for pricing options under incomplete information is due to Föllmer and Sondermann [7], who first proposed the minimization of quadratic risk.

Their work, as well as that of Bouleau and Lamberton, focused on the case when the price of the underlying asset is a martingale. The more general semimartingale case was considered by: Duffie and Richardson [6], Schweizer [18, 19, 20, 21], Hipp [10], Monat and Stricker [13], Schäl [17], and a definitive solution was provided by Schweizer and Rheinländer [16], and Gourieroux, Laurent, and Pham [14], with different methods.

In the meantime, the random behavior of volatility turned out to be a major issue in applied option pricing, and Hull and White [11], Stein and Stein [22] and Heston [9] proposed different models with stochastic volatility. In fact, such models are special cases of incomplete information, and can be effectively embedded in the theoretical framework developed by mathematicians.

A particularly appealing feature of mean-variance hedging is that European options prices are calculated as the expectations of their respective payoff under a (possibly signed) martingale measure $\tilde{P}$, introduced by Schweizer [21]. The optimal strategy can also be found in terms of this measure, therefore it is not surprising that considerable effort has been devoted to its explicit calculation. 
In this paper, we address the problem of calculating $\tilde{P}$ in presence of volatility jumps or, more generally, when the so-called market price of risk $\lambda=\frac{\mu-r}{\sigma}$ follows a possibly discontinuous process.

We consider the following market model, where each state of nature $(\omega, \eta)$ belongs to the product space $(\Omega \times H)$, endowed with the product measure $P^{\Omega} \otimes P^{H}$ :

$$
\left\{\begin{array}{l}
d S_{t}(\omega, \eta)=\mu_{t}(\eta) S_{t}(\omega, \eta) d t+\sigma_{t}(\eta) S_{t}(\omega, \eta) d W_{t}(\omega) \\
B_{t}=\exp \left(\int_{0}^{t} r_{s} d s\right)
\end{array}\right.
$$

and $r$ is a constant.

We assume the existence on $H$ of a set $\mathcal{A}$ of martingales with the representation property: this somewhat technical condition is in fact satisfied in most models present in the literature.

First we characterize martingale measures in terms of $\mathcal{A}$, then, exploiting some results of Schweizer, we isolate the density of the meanvariance optimal martingale measure. When volatility follows a diffusion process (such as in the Heston or Hull and White models, only to mention two of them), this result was already obtained by Laurent and Pham with stochastic control arguments.

It turns out that the jump size distribution is a critical issue: in fact, finite distributions are easily handled by $n$ martingales, where $n$ is the cardinality of the jump size support. On the contrary, an infinite distribution for the jump size requires a more general approach involving random measures.

Moreover we show calculations for sample models where volatility jumps are random both in size and in time of occurrence. For all of them, we calculate the density of the mean-variance optimal measure, and the law of the jumps under $\tilde{P}$.

In the last section, we calculate the mean-variance hedging strategy for a call option, exploiting the change of numeraire technique of El Karoui, Geman and Rochet [8], as well as the general formula in feedback form of Rheinländer and Schweizer [16].

\section{Preliminaries}

For all standard definitions on stochastic processes, we refer to Dellacherie and Meyer [4].

1.1. Stopping Times. We recall here some definitions and properties of stopping times, which will come useful in the sequel. In this subsection, we assume that all random variables are defined on some probability space $(\Omega, \mathcal{F}, P)$. 
Proposition 1.1. Let $\tau$ be a real-valued, Borel random variable, and $\mathcal{F}^{\tau}$ the smallest filtration under which $\tau$ is a stopping time. We have that:

$$
\mathcal{F}_{t}^{\tau}=\tau^{-1}(\mathcal{B}([0, t])) \vee \tau^{-1}(t, \infty)
$$

where $\mathcal{B}(A)$ is the family of Borel subsets of $A$.

Proof. By definition, $\mathcal{F}_{t}^{\tau}=\sigma(\{\tau \leq s\}, s \leq t)$. Since $\mathcal{F}_{t}^{\tau}$ is a $\sigma$-field and contains all sublevels of $\tau$ within $[0, t]$, it necessarily contains the inverse images of all Borel sets of $[0, t]$. The set $\tau^{-1}(t, \infty)$ is the complement of $\tau^{-1}[0, t]$, and belongs to the $\sigma$-field. The reverse inclusion is trivial. Finally, the right-hand side in (2) is easily seen to be a $\sigma$-field.

Remark 1.2. An immediate consequence of Proposition 1.1 is the rightcontinuity of $\mathcal{F}_{t}^{\tau}$. Moreover, $\mathcal{F}_{t-}^{\tau}=\tau^{-1}(\mathcal{B}([0, t))) \vee \tau^{-1}[t, \infty)$. This means that the augmentation of $\mathcal{F}_{t}^{\tau}$ is continuous if and only if the law of $\tau$ is diffuse.

Corollary 1.3. The filtration generated by the random variable $\tau \wedge t$ coincides with $\mathcal{F}^{\tau}$ if and only if $\tau$ is an optional time, that is, if $\{\tau<$ $t\} \in \mathcal{F}_{t}$ for all $t$.

Definition 1.4. A stopping time $\tau$ is totally inaccessible if it is strictly positive and for every increasing sequence of stopping times $\tau_{1}, \ldots, \tau_{n}$, such that $\tau_{n}<\tau$ for all $n, P\left(\lim _{n \rightarrow \infty} \tau_{n}=\tau, \tau<\infty\right)=0$.

Totally inaccessible stopping times can be characterized as follows:

Proposition 1.5. Let $\tau$ a strictly positive stopping time. The following properties are equivalent:

i) $\tau$ is totally inaccessible;

ii) There exists a uniformly integrable martingale $M_{t}$, continuous outside the graph of $\tau$ such that $M_{0}=0$ and $\Delta M_{\tau}=1$ on $\{\tau<\infty\}$.

Proof. See Dellacherie and Meyer [5], VI.78.

Proposition 1.6. If the law of a stopping time is diffuse, then it is totally inaccessible with respect to $\mathcal{F}^{\tau}$.

Proof. See Dellacherie and Meyer [4], IV.107.

Definition 1.7. Let $A$ be an adapted process, with $A_{0}=0$ and locally integrable variation. The compensator of $A$ is defined as the unique predictable process $\tilde{A}$ such that $A-\tilde{A}$ is a local martingale.

Remark 1.8. In particular, the compensator of an increasing process is itself an increasing predictable process.

Proposition 1.9. Let $\tau$ be a stopping time with a diffuse law. Then the compensator of the process $1_{\{\tau \leq t\}}$ with respect to $\mathcal{F}^{\tau}$ is given by

$$
\tilde{A}_{t}=-\log \left(1-F_{t \wedge \tau}\right)
$$

where $F_{x}=P(\tau \leq x)$. 
Proof. By assumption, $M_{t}=1_{\{\tau \leq t\}}-\tilde{A}_{t}$ is a local martingale, and by Remark 1.8, $\tilde{A}_{t}$ is an increasing process. Therefore we have:

$$
\sup _{s \leq t} M_{s} \leq 1
$$

and $M_{t}$ is in fact a martingale (see for instance Protter, page 35, Theorem 47). We look for a compensator of the form $\tilde{A}_{t}=a_{t \wedge \tau}$, with $a: \mathbb{R}^{+} \mapsto \mathbb{R}^{+}$. Hence:

$$
E\left[1_{\{\tau \leq s\}}-1_{\{\tau \leq t\}} \mid \mathcal{F}_{t}\right]=E\left[a_{s \wedge \tau}-a_{t \wedge \tau} \mid \mathcal{F}_{t}\right] \quad \forall s \geq t
$$

It follows that:

$$
\frac{P(t<\tau \leq s)}{P(t<\tau)}=\frac{E\left[1_{\{t<\tau \leq s\}}\left(a_{\tau}-a_{t}\right)+1_{\{t<\tau\}}\left(a_{s}-a_{t}\right)\right]}{P(t<\tau)}
$$

This equality can be rewritten as an integral equation:

$$
F_{s}-F_{t}=\int_{t}^{s}\left(a_{x}-a_{t}\right) d F_{x}+\int_{s}^{\infty}\left(a_{s}-a_{t}\right) d F_{x}
$$

It is easy to check by substitution that the unique solution to this integral equation is given by $a_{x}=-\log \left(1-F_{x}\right)$. By the uniqueness, this is the only compensator of $1_{\{\tau \leq t\}}$.

1.2. Martingale Representation. We denote by $\mathbf{M}^{2}$ the set of squareintegrable martingales on a probability space $(\Omega, \mathcal{F}, P)$ endowed with a filtration $\mathcal{F}_{t}$ satisfying the usual hypotheses.

Definition 1.10. A set $\mathcal{A}=\left\{X^{1}, \ldots X^{n}\right\} \subset \mathbf{M}^{2}$ is strongly orthogonal if $X_{i} X_{j} \in \mathbf{M}^{2}$ for $i, j \in\{1 \ldots n\}$.

Definition 1.11. A set $\mathcal{A}$ of strongly orthogonal martingales $X^{1}, \ldots, X^{n}$ has the representation property if $\mathcal{I}=\mathbf{M}^{2}$, where

$$
\mathcal{I}=\left\{X: X_{t}=\sum_{i=1}^{n} \int_{0}^{t} H_{s}^{i} d X_{s}^{i}\right\}
$$

for all $H$ predictable such that

$$
E\left[\int_{0}^{t}\left(H_{s}^{i}\right)^{2} d\left[X^{i}\right]_{s}\right]<\infty \quad 1 \leq i \leq n \quad \forall t
$$

In this paper we will need the following result:

Proposition 1.12. Let $\mathcal{A}=\left(X^{1}, \ldots, X^{n}\right)$ a set of strongly orthogonal martingales. If there is only a martingale measure for $\mathcal{A}$, then $\mathcal{A}$ has the representation property.

Proof. See Dellacherie and Meyer [5], VIII.58 or Protter [15], IV.37. 
1.3. Random measures. For completeness, we provide some basic definitions on random measures: we refer to [12] for a complete treatment of the subject.

Let $(H, \mathcal{F}, P)$ be a probability space.

Definition 1.13. A random measure on $\mathbb{R}_{+} \times \mathbb{R}$ is a family $\nu=$ $(\nu(\eta ; d t, d x): \eta \in H)$ of nonnegative measures on $\left(\mathbb{R}_{+} \times \mathbb{R}, \mathcal{B}\left(\mathbb{R}_{+}\right) \otimes\right.$ $\mathcal{B}(\mathbb{R}))$, satisfying $\nu(\eta ;\{0\} \times \mathbb{R})=0$ identically.

We set $\tilde{H}=H \times \mathbb{R}_{+} \times \mathbb{R}$, with $\sigma$-fields $\tilde{\mathcal{O}}=\mathcal{O} \otimes \mathcal{B}(\mathbb{R})$ and $\tilde{\mathcal{P}}=$ $\mathcal{P} \otimes \mathcal{B}(\mathbb{R})$, where $\mathcal{O}$ and $\mathcal{P}$ are respectively the optional and predictable $\sigma$-field on $H \times \mathbb{R}_{+}$.

Definition 1.14. For any $\tilde{O}$-measurable function $h(\eta ; t, x)$ we define the integral process $h * \nu$ as

$h * \nu_{t}(\eta)= \begin{cases}\int_{[0, t] \times H} h(\eta, s, x) \nu(\eta ; d s, d x) & \text { if } \int_{[0, t] \times H}|h(\eta, s, x)| \nu(\eta ; d s, d x)<\infty \\ +\infty & \text { otherwise }\end{cases}$

Analogously, a random measure $\nu$ is optional (resp. predictable) if the process $h * \nu$ is optional (resp. predictable) for every optional (resp. predictable) function $h$.

Definition 1.15. An integer-valued random measure is a random measure that satisfies:

i) $\nu(\eta ;\{t\} \times \mathbb{R}) \leq 1$ identically;

ii) for each $A \in \mathcal{B}\left(\mathbb{R}_{+}\right) \otimes \mathcal{B}(\mathbb{R}), \nu(\cdot, A)$ take its values in $\overline{\mathbb{N}}$;

iii) $\nu$ is optional;

iv) $\tilde{\mathcal{P}}$ - $\sigma$-finite, i.e. there exists a $\tilde{\mathcal{P}}$-measurable partition $A_{n}$ of $\tilde{H}$ such that $1_{A_{n}} * \nu_{\infty}$ is integrable.

By proposition 1.14 of [12] we obtain the following characterization of integer-valued random measures.

Proposition 1.16. If $\nu$ is an integer-valued random measure, there exists a random set $D=\bigcup\left[T_{n}\right]$, where $\left(\left[T_{n}\right]\right)_{n \in \mathbb{N}}$ is the sequence of graphs of stopping times $T_{n}$, and a real optional process $\beta$ such that

$$
\nu(\eta ; d t, d u)=\Sigma_{s \geq 0} 1_{D}(\eta, s) \epsilon_{\left(s, \beta_{s}(\eta)\right)}(d t, d u)
$$

If $\left(T_{n}\right)_{n \in \mathbb{N}}$ satisfies $\left[T_{n}\right] \cap\left[T_{m}\right]=$ for $n \neq m$, it is called an exhausting sequence for $D$.

Proposition 1.17. Let $X$ be an adapted càdlàg $\mathbb{R}^{d}$-valued process. Then:

$$
\nu^{X}(\eta ; d t, d x)=\sum_{s \geq 0} 1_{\left\{\Delta X_{s} \neq 0\right\}} \epsilon_{\left\{s, \Delta X_{s}(\eta)\right\}}(d t, d x)
$$

defines an integer-valued random measure on $\mathbb{R}_{+} \times \mathbb{R}^{d}$. 
Proof. See [12], Chapter II, Proposition 1.16.

Definition 1.18. A multivariate point process is an integer-valued random measure $\nu$ on $\mathbb{R}_{+} \times \mathbb{R}$ such that $\nu(\eta ;[0, t] \times \mathbb{R})<\infty$ for all $\eta, t \in \mathbb{R}_{+}$.

Proposition 1.19. Let $\nu$ be an optional $\tilde{\mathcal{P}}-\sigma$-finite random measure. There exists a random measure $\nu^{p}$, called compensator of $\nu$, and unique up to a P-null set, such that:

i) $\nu^{p}$ is a predictable random measure;

ii) $E\left[h * \nu_{\infty}^{p}\right]=E\left[h * \nu_{\infty}\right]$ for every nonnegative $\tilde{P}$-measurable function $h$ on $\tilde{\Omega}$.

Also, if $|h| * \nu$ is increasing and locally integrable, then $h *\left(\nu-\nu^{p}\right)$ is a local martingale.

Proof. See Theorem [12], Chapter II, Theorem 1.8.

Let $\nu=\Sigma_{s \geq 0} 1_{D}(\eta, s) \epsilon_{\left(s, \beta_{s}(\eta)\right)}(d t, d u)$ be an integer random measure and $\nu^{p}$ its compensator.

\section{Definition 1.20.}

1. We denote by $G_{l o c}(\nu)$ the set of all $\tilde{\mathcal{P}}$-measurable real-valued function $h$ such that the process

$$
\tilde{h}_{t}(\eta)=h(\eta, t, \beta(\eta)) 1_{D}(\eta, t)-\int_{H} h(\eta, s, x) \nu(\eta ; s \times d x)
$$

has the property that $\left[\Sigma_{s \leq .}\left(\tilde{h}_{s}\right)^{2}\right]^{\frac{1}{2}}$ is locally integrable and increasing.

2. If $h \in G_{l o c}(\nu)$ we call stochastic integral with respect to $\nu-\nu^{p}$ and denote by $h *\left(\nu-\nu^{p}\right)$ any purely discontinuous local martingale $X$ such that $\tilde{h}$ and $\Delta X$ are indistinguishable.

Definition 1.21. A compensated random measure $\nu-\nu^{p}$ has the representation property on $H$ iff every square integrable martingale $M_{t}$ on $\left(H, \mathcal{F}^{H}, P^{H}\right)$ can be written as

$$
M_{t}=M_{0}+k *\left(\nu-\nu^{p}\right)_{t}
$$

for some $\tilde{\mathcal{P}}$-measurable process $k$.

We recall that from proposition 1.28, first chapter of [12] follows that $h *\left(\nu-\nu^{p}\right)_{t}=h * \nu_{t}-h * \nu_{t}^{p}$ for every $\tilde{\mathcal{P}}$-measurable process $h$ such that $|h| * \nu_{t}$ is a locally integrable increasing process. Consequently, we obtain in theorem 1.21 that the integral $h *\left(\nu-\nu^{p}\right)$, which is a-priori stochastic, coincides with the Stieltjes integral $h * \nu-h * \nu^{p}$. It follows that every local martingale on $H$ has finite variation.

Theorem 1.22. Assume that $\nu$ is a multivariate point process. Then, $\nu-\nu^{p}$ has the representation property with respect to the smallest filtration under which $\nu$ is optional.

Proof. See theorem 4.37 of [12]. 


\section{The Market Model}

We introduce here a simple model for a market with incomplete information.

We have two complete filtered probability spaces: $\left(\Omega, \mathcal{F}^{\Omega}, \mathcal{F}_{t}^{\Omega}, P^{\Omega}\right)$ and $\left(H, \mathcal{F}^{H}, \mathcal{F}_{t}^{H}, P^{H}\right)$. We denote by $W_{t}$ a standard Brownian Motion on $\Omega$, and assume that $\mathcal{F}_{t}^{\Omega}$ is the $P^{\Omega} \otimes \boldsymbol{q}^{H}$-augmentation of the filtration generated by $W$. Our set of states of nature is given by the product space $\left(\Omega \times H, \mathcal{F}^{\Omega} \otimes \mathcal{F}^{H}, P^{\Omega} \otimes P^{H}\right)$.

We have a risk-free asset $B_{t}$ and a discounted risky asset $X_{t}=\frac{S_{t}}{B_{t}}$, with the following dynamics:

$$
\left\{\begin{array}{l}
d X_{t}(\omega, \eta)=\left(\mu_{t}(\eta)-r_{t}\right) X_{t}(\omega, \eta) d t+\sigma_{t}(\eta) X_{t}(\omega, \eta) d W_{t}(\omega) \\
B_{t}=\exp \left(\int_{0}^{t} r_{s} d s\right)
\end{array}\right.
$$

where $r$ is a deterministic function of time. We assume that the equation for $X$ admits $P^{H}$-a.e. a unique strong solution with respect to the filtration $\mathcal{F}^{W}$ or, equivalently, that there exists a unique strong solution with respect to the filtration $\tilde{\mathcal{F}}_{t}=\mathcal{F}_{t}^{W} \otimes \mathcal{F}^{H}$. This is satisfied under fairly weak assumptions: for example, it is sufficient that $\mu$ and $\sigma$ are $P^{H}$-a.e. bounded. Denoting by $\mathcal{F}^{X}$ the filtration generated by $X$, we also assume that $\mathcal{F}_{t}^{X}=\mathcal{F}_{t}^{W} \otimes \mathcal{F}_{t}^{H}$. In particular, at time $T$ all information is revealed through the observation of the process $X$.

The following proposition helps checking whether this condition is satisfied:

Proposition 2.1. Let $X_{t}$ be defined as in (3). Then, if

i) $\mu_{t}$ is $\mathcal{F}_{t}^{X}$-measurable,

ii) $\mathcal{F}_{t}^{H} \subset \mathcal{F}_{t}^{X}$.

then $\mathcal{F}_{t}^{X}=\mathcal{F}_{t}^{W} \otimes \mathcal{F}_{t}^{H}$.

Proof. By definition of $X_{t}$, we immediately have that $\mathcal{F}_{t}^{X} \subset \mathcal{F}_{t}^{\Omega} \otimes \mathcal{F}_{t}^{H}$. To see that the reverse inclusion holds, observe first that by (3):

$$
W_{t}=-\int_{0}^{t} \frac{\mu_{s}}{\sigma_{s}} d s+\int_{0}^{t} \frac{d X_{s}}{\sigma_{s} X_{s}}
$$

By $i$ ), the first term above is $\mathcal{F}_{t}^{X}$-measurable. For the second, note that

$$
\int_{0}^{t} \sigma(s, \eta)^{2} X_{s}^{2} d s=\lim _{\sup _{i}\left|t_{i+1}-t_{i}\right| \rightarrow 0} \sum_{i=1}^{n}\left|X_{t_{i+1}}-X_{t_{i}}\right|^{2}
$$

where the limit holds in probability, uniformly in $t$. This proves that $\mathcal{F}_{t}^{\Omega} \subset \mathcal{F}_{t}^{X}$, and by $\left.i i\right)$ the proof is complete.

A simpler version of this model was introduced by Delbaen and Schachermayer [3], while it can be found in the above form in Pham, Rheinländer, and Schweizer [14]. 
In this framework, we study the problem of an agent wishing to hedge a certain European option $H\left(X_{T}\right)$ expiring at a fixed time $T$. Hedging performance is defined as the $L^{2}$-norm of the difference, at expiration, between the liability and the hedging portfolio. More precisely, we look for a solution to the minimization problem:

$$
\min _{\substack{c \in \mathbb{R} \\ \theta \in \Theta}} E\left[\left(H\left(X_{T}\right)-c-G_{T}(\theta)\right)^{2}\right]
$$

where

$$
G_{t}(\theta)=\int_{0}^{t} \theta_{s} d X_{s} \quad \text { and } \quad \Theta=\left\{\theta \in L(X), G_{t}(\theta) \in \mathcal{S}^{2}(P)\right\}
$$

Here $L(X)$ denotes the space of $X$-integrable predictable processes, and $\mathcal{S}^{2}$ the space of semimartingales $Y$ decomposable as $Y=Y_{0}+M+A$, where $M$ is a square-integrable martingale, and $A$ is a process of squareintegrable variation.

This problem is generally nontrivial, since the agent has not access to the filtration $\tilde{\mathcal{F}}$, but only to $\mathcal{F}^{X}$. Indeed, Reihnländer and Schweizer [16] and, independently, Gourieroux, Laurent and Pham [14], proved that problem (5) admits a unique solution for all $H \in L^{2}(P)$, under the standing hypothesis:

$$
G_{T}(\Theta) \text { is closed }
$$

Moreover, in [21] it is shown that the option price, (i.e. the optimal value for $c$ ), and the optimal hedging strategy $\theta$ can be computed in terms of $\tilde{P}$, the variance-optimal martingale measure. In fact, the option price is given by $c=\tilde{E}[H]$.

Definition 2.2. We define the sets of signed martingale measures $\mathcal{M}_{s}^{2}$, and of equivalent martingale measures $\mathcal{M}_{e}^{2}$ :

$$
\begin{aligned}
& \mathcal{M}_{s}^{2}=\left\{Q \ll P: \frac{d Q}{d P} \in L^{2}(P), X_{t} \text { is a } Q \text {-local martingale }\right\} \\
& \mathcal{M}_{e}^{2}=\left\{Q \in \mathcal{M}_{s}^{2}: Q \sim P:\right\}
\end{aligned}
$$

Definition 2.3. The variance optimal martingale measure is the unique solution $\tilde{P}$ (if it exists) to the minimum problem:

$$
\min _{Q \in \mathcal{M}_{s}^{2}} E\left[\left(\frac{d Q}{d P}\right)^{2}\right]
$$

If $\mathcal{M}_{s}^{2}$ is nonempty, then $\tilde{P}$ always exists, as it is the minimizer of the norm in a convex set: the problem is that it may not be positive definite, thereby leading to (possibly) negative option prices. However, if $X_{t}$ has continuous paths, and under the standard assumption

$$
\mathcal{M}_{e}^{2} \neq \emptyset
$$


Delbaen and Schachermayer [3] have shown that $\tilde{P} \in \mathcal{M}_{e}^{2}$. Since we are dealing with continuous processes, and we will always assume (NA), we need not worry about this issue.

In this paper, using a representation formula from [1], we compute explicitly $\tilde{P}$ for some sample models.

\section{Representation of Martingale Measures}

Here we summarize some of the main results of [1] in order to show that the set $\mathcal{M}_{e}^{2}$ of equivalent martingale measures admits a convenient representation. We denote by $\lambda_{t}=\frac{\mu_{t}-r_{t}}{\sigma_{t}}$ the so-called market price of risk.

Theorem 3.1. If there exists a n-dimensional martingale $M$ on $H$ such that:

i) $\left[M_{i}, M_{j}\right] \equiv 0$ for all $i \neq j$;

ii) $M$ has the representation property for $\mathcal{F}_{t}^{H}$;

then we have for every $Q \in \mathcal{M}_{e}^{2}$ :

$$
\frac{d Q}{d P}=\mathcal{E}\left(-\int_{0}^{\cdot} \lambda_{t}(\eta) d W_{t}\right)_{T} \mathcal{E}\left(\int_{0}^{\cdot} k_{t}(\omega, \eta) d M_{t}\right)_{T}
$$

where $k_{t}$ is such that $\mathcal{E}\left(-\int_{0}^{\cdot} \lambda_{t}(\eta) d W_{t}\right)_{t} \mathcal{E}\left(\int_{0}^{\cdot} k_{t}(\omega, \eta) d M_{t}\right)_{t}$ is a square integrable martingale and $k_{t} \cdot \Delta M_{t}>-1$.

By Theorem 3.1, a martingale measure is uniquely determined by the process $k$ which appears in its representation. In particular, $k=$ 0 corresponds to the minimal martingale measure $\hat{P}$ introduced by Föllmer and Schweizer [7].

The next proposition identifies $\tilde{P}$.

Proposition 3.2. In the same assumptions as Theorem 3.1, we have:

$$
\frac{d \tilde{P}}{d P}=\mathcal{E}\left(-\int_{0}^{\cdot} \lambda_{t} d W_{t}\right)_{T} \mathcal{E}\left(\int_{0}^{\cdot} \tilde{k}_{t}(\eta) d M_{t}\right)_{T}
$$

where $\tilde{k}_{t}$ is a solution of the following equation

$$
\mathcal{E}\left(\int_{0}^{\cdot} \tilde{k}_{t}(\eta) d M_{t}\right)_{T}=\frac{\exp \left(-\int_{0}^{T} \lambda_{t}^{2}(\eta) d t\right)}{E\left[\exp \left(-\int_{0}^{T} \lambda_{t}^{2}(\eta) d t\right)\right]}
$$

such that $\mathcal{E}\left(-\int_{0}^{\cdot} \lambda_{t} d W_{t}\right)_{t} \mathcal{E}\left(\int_{0}^{\cdot} \tilde{k}_{t}(\eta) d M_{t}\right)_{t}$ is a square integrable martingale.

Remark 3.3. Proposition 3.2 shows how the change of measure works on $\Omega$ and $H$. In fact, provided that $\hat{P}$ exists, we can write:

$$
\frac{d \tilde{P}}{d P}=\frac{d \tilde{P}}{d \hat{P}} \cdot \frac{d \hat{P}}{d P}
$$


where

$$
\frac{d \hat{P}}{d P}=\mathcal{E}\left(-\int_{0}^{T} \lambda_{t} d W_{t}\right) \quad \text { and } \quad \frac{d \tilde{P}}{d \hat{P}}=\frac{\exp \left(-\int_{0}^{T} \lambda_{t}^{2}(\eta) d t\right)}{E\left[\exp \left(-\int_{0}^{T} \lambda_{t}^{2}(\eta) d t\right)\right]}
$$

Since in our model $\frac{d \tilde{P}}{d \hat{P}}$ does not depend on $\omega$, we have:

$$
\frac{d \tilde{P}_{H}}{d P_{H}}=E\left[\frac{d \tilde{P}}{d P} \mid \mathcal{F}_{H}\right]=E\left[\frac{d \tilde{P}}{d \hat{P}} \cdot \frac{d \hat{P}}{d P} \mid \mathcal{F}_{H}\right]=\frac{d \tilde{P}}{d \hat{P}} E\left[\frac{d \hat{P}}{d P} \mid \mathcal{F}_{H}\right]=\frac{d \tilde{P}}{d \hat{P}}
$$

This provides a rule of thumb for changing measure from $P$ to $\tilde{P}$ via $\hat{P}$. First change $P$ to $\hat{P}$ by a direct use of Girsanov theorem: this amounts to replacing $\mu$ with $r$ in (3), and is the key of risk-neutral valuation. $P_{H}$ is not affected by this step.

In principle, one could repeat the same argument from $\hat{P}$ to $\tilde{P}$, but this involves calculating the $\tilde{k}_{t}(\eta)$. As we show with an example in the last section, this task may prove hard even in simple cases.

A more viable alternative is calculating $\tilde{P}_{H}$ with the above formula. This avoids dealing with $\tilde{k}$ directly, although its existence is still needed through Proposition 3.2.

A sufficient condition for the existence of $\hat{P}$ is the Novikov condition, namely:

$$
E\left[\exp \left(\frac{1}{2} \int_{0}^{T} \lambda_{t}^{2} d t\right)\right]<\infty
$$

and is satisfied by all the examples in the last section.

\section{Volatility JUmps And RAndom MEAsures}

Although most markets models considered in the literature can be embedded in a framework consistent with Theorem 3.1, there are some remarkable exceptions. For example, continuously distributed jumps in volatility can generate filtrations where no finite set of martingales has the representation property (see the examples in the next section).

In these cases, we can still represent martingales in terms of integrals with respect to a compensated random measure $\nu-\nu^{p}$, thereby obtaining an analogous of Theorem 3.1 and Proposition 3.2.

Note that the following results are complementary to those in the previous section, but do not directly generalize them: in fact any model with volatility following a diffusion process is covered in the previous section, and not in the present one. 
Theorem 4.1. If there exists a compensated, integer-valued, random measure $\nu-\nu^{p}$ on $E \times \mathbb{R}_{+} \times \mathbb{R}$ such that:

i) $\mathcal{F}^{H}$ coincides with the smallest filtration under which $\nu$ is optional;

ii) $\nu-\nu^{p}$ has the representation property on $\left(H, \mathcal{F}^{H}, P^{H}\right)$.

Then we have for every $Q \in \mathcal{M}_{e}^{2}$ :

$$
\frac{d Q}{d P}=\mathcal{E}\left(-\int_{0}^{\cdot} \lambda_{t} d W_{t}\right)_{T} \mathcal{E}\left(k *\left(\nu-\nu^{p}\right)\right)_{T}
$$

where $k_{t}$ is such that $\Delta(k * \nu)_{t}>-1$ and $\mathcal{E}\left(-\int_{0}^{\cdot} \lambda_{t} d W_{t}\right)_{t} \mathcal{E}\left(k *\left(\nu-\nu^{p}\right)\right)_{t}$ is a square integrable martingale.

As in the previous section, we need these lemmas:

Lemma 4.2. In the same assumptions as Theorem 4.1, every square integrable martingale $M_{t}$ on the space $\left(\Omega \times E, \mathcal{F}^{\Omega} \otimes \mathcal{F}^{H}, P^{\Omega} \otimes P^{H}\right)$ with respect to $\mathcal{F}_{t}$ can be written as

$$
M_{t}=M_{0}+\int_{0}^{t} h_{s} d W_{s}+k *\left(\nu-\nu^{p}\right)_{t}
$$

Proof. Let us denote the set of martingales for which the thesis hold by $\mathfrak{M}$. We want to show that $\mathfrak{M}=M^{2}(\Omega \times H)$.

By representation property, every square integrable martingale $M_{t}(\omega)$ on $\Omega \times H$ depending only on $\omega$ belongs to $\mathfrak{M}$, since it can be written as $M_{t}=M_{0}+\int_{0}^{t} h_{s} d W_{s}$. Analogously, every $N_{t}(\eta)$ belongs to $\mathfrak{M}$, since it is of the form $N_{t}=N_{0}+k *\left(\nu-\nu^{p}\right)_{t}$, where $k$ is a $\tilde{\mathcal{P}}$-measurable process and $|k| * \nu_{t}$ is locally integrable.

Denoting $P_{t}(\omega, \eta)=N_{t}(\eta) M_{t}(\omega)$, we have that $P_{t}$ is a square integrable martingale on $\Omega \times H$. Setting $P_{t}=P_{t}^{d}+P_{t}^{c}$, where $P_{t}^{c}$ and $P_{t}^{d}$ are the continuous and purely discontinuous parts of $P$, we have that $\Delta P_{t}=\Delta P_{t}^{d}=M_{t} \Delta N_{t}$. By 1.20, it follows that $P_{t}^{d}=$ $P_{0}^{d}+(M k) *\left(\nu-\nu^{p}\right)_{t}$. Also, by Itô's formula, $P_{t}^{c}=P_{0}^{c}+\int_{0}^{t} N_{s} h_{s} d W_{s}$. This shows that any linear combination $\sum_{i} M_{i}(\omega) N_{i}(\eta)$ belongs to $\mathfrak{M}$ and, by a monotone class argument, it is easy to see that $\mathfrak{M}$ is dense in $\mathbf{M}^{2}$. Hence, for every $Z_{t} \in M^{2}$ there exist a sequence $X_{t}^{n}$ of squareintegrable martingales such that $X_{t}^{n}=X_{0}+\int_{0}^{t} h_{s}^{n} d W_{s}+k^{n} *\left(\nu-\nu^{p}\right)_{t}$. By the identity:

$$
E\left[X_{T}^{n}\right]=E\left[\int_{0}^{T}\left(h_{s}^{n}\right)^{2} d s\right]+E\left[\left(k_{s}^{n}\right)^{2} * \nu_{s}^{p}\right]
$$

it follows that $h^{n}$ and $k^{n}$ are Cauchy sequences respectively in

$$
\begin{gathered}
\left\{h_{t}(\eta) \text { predictable: } E\left[\int_{0}^{T} h_{s}^{2} d s\right]<\infty\right\} \quad \text { and } \\
\left\{k_{t}(\eta, x) \text { predictable: } E\left[k_{s}^{2} * \nu_{s}^{p}\right]<\infty\right\}
\end{gathered}
$$

Since these spaces are complete, the proof is finished. 
Lemma 4.3. Let $Z_{T}$ be a strictly positive, square-integrable random variable and denote $Z_{t}=E\left[Z_{T} \mid \mathcal{F}_{t}\right]$. Then, if $Z_{t}=Z_{0}+H *\left(\nu-\nu^{p}\right)_{t}$, we have:

$$
Z_{t}=Z_{0} \mathcal{E}\left(\frac{H}{Z_{-}} *\left(\nu-\nu^{p}\right)_{t}\right) .
$$

Proof. If there exists a martingale $M_{t}=M_{0}+K *\left(\nu-\nu^{P}\right)_{t}$ such that $Z_{t}=Z_{0} \mathcal{E}\left(M_{t}\right)$, then it is unique. In fact, if $N_{t}=N_{0}+H *\left(\nu-\nu^{P}\right)_{t}$ and $Z_{t}=Z_{0} \mathcal{E}\left(N_{t}\right)$, we immediately have $\Delta M_{t}=\Delta N_{t}$. Since $M_{t}$ and $N_{t}$ are purely discontinuous martingales by definition 1.20 , they must coincide up to evanescent sets.

In particular, we have that $\Delta M_{t}=\frac{\Delta Z_{t}}{Z_{t-}}$. For all $t>0, \frac{\Delta Z_{t}}{Z_{t-}}$ coincides with the jumps of the purely discontinuous martingale $\frac{H}{Z-} *\left(\nu-\nu^{P}\right)_{t}$, therefore $M_{t}$ exists and is given by $M_{t}=\log \left(Z_{0}\right)+\frac{H}{Z-} *\left(\nu-\nu^{P}\right)_{t}$.

Proposition 4.4. In the same assumptions as Theorem 4.1, we have:

$$
\frac{d \tilde{P}}{d P}=\mathcal{E}\left(-\int_{0} \lambda_{t} d W_{t}\right)_{T} \mathcal{E}\left(\tilde{k} *\left(\nu-\nu^{p}\right)\right)_{T}
$$

where $\tilde{k}_{t}$ is a solution of the following equation

$$
\mathcal{E}\left(\tilde{k} *\left(\nu-\nu^{p}\right)\right)_{T}=\frac{\exp \left(-\int_{0}^{T} \lambda_{t}^{2}(\eta) d t\right)}{E\left[\exp \left(-\int_{0}^{T} \lambda_{t}^{2}(\eta) d t\right)\right]}
$$

such that $\mathcal{E}\left(-\int_{0} \lambda_{t} d W_{t}\right)_{t} \mathcal{E}\left(\tilde{k} *\left(\nu-\nu^{p}\right)\right)_{t}$ is a square integrable martingale.

Proof. The proof is formally analogous to that of Theorem 1.16 of [1], by the previous results and the representation property of the compensated random measure $\nu-\nu^{p}$.

\section{EXAMPles}

We now show how the results in the previous sections provide convenient tools for calculating $\tilde{P}$ (and thus pricing options) in models where volatility jumps. We start with a simple model where jumps occur at fixed times, and can take only two values. We then discuss the more general cases of jumps occurring at stopping times, and with arbitrary distributions.

5.1. Deterministic volatility jumps. In discrete-time fashion, the following model was introduced in RiskMetrics Monitor [23] as an improvement of the standard lognormal model for calculating Value at Risk.

We set $H=\{0,1\}^{n}$ and denote $\eta=\left\{a_{1}, \ldots, a_{n}\right\} . a_{1} \ldots, a_{n}$ are Bernoulli IID random variable, so that $H$ is endowed with the product 
measure from $\{0,1\} . \mathcal{F}_{t}^{H}$ contains all information on jumps up to time $t$, therefore it is equal to the parts of $\left\{a_{i}\right\}_{t_{i} \leq t}$. Setting $t_{i}=i \frac{T}{n+1}$, the dynamics of $\mu$ and $\sigma$ is given by:

$$
\left\{\begin{array}{l}
\mu_{t}=1_{\left\{0 \leq t<t_{1}\right\}} \mu+\sum_{i=1}^{n} 1_{\left\{t_{i} \leq t<t_{i+1}\right\}} \mu_{a_{i}} \\
\sigma_{t}=1_{\left\{0 \leq t<t_{1}\right\}} \sigma+\sum_{i=1}^{n} 1_{\left\{t_{i} \leq t<t_{i+1}\right\}} \sigma_{a_{i}}
\end{array}\right.
$$

In fact, all we need for mean-variance hedging is the dynamics for $\lambda$ :

$$
\lambda_{t}^{2}=\lambda^{2}+\sum_{i=1}^{n} 1_{\left\{t_{i} \leq t<t_{i+1}\right\}} \lambda_{a_{i}}^{2}
$$

It is easy to check that a martingale with the representation property on $H$ is given by: $M_{t}=\sum_{t_{i}<t}\left(1_{\left\{a_{i}=0\right\}}-p\right)$, where $p=P\left(a_{1}=0\right)$.

We are now ready to see how the change of measure works: in fact, by Theorem 3.1, we have that:

$$
\frac{d \tilde{P}}{d \hat{P}}=\frac{d \tilde{P}_{H}}{d P_{H}}=\frac{\exp \left(-\frac{\lambda_{1}^{2} T}{n}\right)^{a_{1}+\cdots+a_{n}} \exp \left(-\frac{\lambda_{0}^{2} T}{n}\right)^{n-\left(a_{1}+\cdots+a_{n}\right)}}{\left(p \exp \left(-\frac{\lambda_{0}^{2} T}{n}\right)+(1-p) \exp \left(-\frac{\lambda_{1}^{2} T}{n}\right)\right)^{n}}
$$

Since the density above can be written as:

$$
\frac{d \tilde{P}_{H}}{d P_{H}}=\prod_{i=1}^{n} \frac{\exp \left(-a_{i} \frac{\lambda_{1}^{2} T}{n}-\left(1-a_{i}\right) \frac{\lambda_{0}^{2} T}{n}\right)}{\left(p \exp \left(-\frac{\lambda_{0}^{2} T}{n}\right)+(1-p) \exp \left(-\frac{\lambda_{1}^{2} T}{n}\right)\right)^{n}}
$$

it follows that under $\tilde{P}$ the variables $a_{1}, \ldots, a_{n}$ are still independent, and $p$ is replaced by:

$$
\tilde{p}=p \frac{\exp \left(-\frac{\lambda_{1}^{2} T}{n}\right)}{p \exp \left(-\frac{\lambda_{0}^{2} T}{n}\right)+(1-p) \exp \left(-\frac{\lambda_{1}^{2} T}{n}\right)}
$$

5.2. Random volatility jumps. Consider the following model, where $\mu$ and $\sigma$ are constant, until some unexpected event occurs. In other words:

$$
\left\{\begin{array}{l}
\mu_{t}=\mu_{1} 1_{\{t<\tau\}}+\mu_{2} 1_{\{t \geq \tau\}} \\
\sigma_{t}=\sigma_{1} 1_{\{t<\tau\}}+\sigma_{2} 1_{\{t \geq \tau\}}
\end{array}\right.
$$

In fact, all we need is the dynamics for $\lambda$ :

$$
\lambda_{t}^{2}=\lambda_{1}^{2}+\alpha 1_{\{t \geq \tau\}}
$$

where $\alpha=\lambda_{2}^{2}-\lambda_{1}^{2}$. The event $\tau$ which triggers the jump is a totally inaccessible stopping time. That is to say, any attempt to predict it by means of previous information is deemed to failure. $\alpha$ represents the jump size, and it may be deterministic or random. We now solve the problem in three cases: $\alpha$ deterministic, $\alpha$ Bernoulli, and $\alpha$ continuously distributed. 
5.2.1. $\alpha$ deterministic. Since our goal is to find the variance-optimal martingale measure, we start exhibiting a martingale with the representation property for $\mathcal{F}_{H}$, which in this case is the filtration generated by $\tau$.

Proposition 5.1. Let $\tau$ be a stopping time with a diffuse law, and $\tilde{A}$ the compensator of $1_{\{\tau \leq t\}}$. Then the martingale $M_{t}=1_{\{\tau \leq t\}}-\tilde{A}_{t}$ has the representation property.

Proof. Let $Q$ be a martingale measure for $M$, and $\tilde{A}^{Q}$ the compensator of $1_{\{\tau \leq t\}}$ in $Q$. Both $M_{t}$ and $1_{\{\tau \leq t\}}-\tilde{A}^{Q}$ are $Q$-martingales, therefore their difference $\tilde{A}^{Q}-\tilde{A}$ is also a martingale. However, it is also a finite variation process, therefore it must be identically zero.

Since $\widetilde{A}^{Q}=\tilde{A}$, by Proposition 1.9, the c.d.f.'s of $\tau$ under $P$ and $Q$ are equal. This implies that $Q=P, \mathcal{F}^{\tau}$-a.e. Theorem 1.12 concludes the proof.

We now compute $P_{H}$, that is the law of $\tau$ under $\tilde{P}$. For simplicity, assume that $\tau$ has a density, and denote it by $f_{t}$. We have:

$$
\begin{aligned}
\tilde{f}_{t}= & \frac{d \tilde{P}}{d \hat{P}} f_{t}= \\
& =\frac{\exp \left(-\int_{0}^{T} \lambda_{s}^{2}(\eta) d s\right)}{c} f_{t}= \begin{cases}\frac{1}{c} \exp \left(-\lambda_{1} T+\alpha t\right) f_{t} & \text { if } t<T \\
\frac{1}{c} \exp \left(-\lambda_{1} T\right) f_{T} & \text { if } t \geq T\end{cases}
\end{aligned}
$$

where $c=E\left[\exp \left(-\int_{0}^{T} \lambda_{s}^{2}(\eta) d s\right)\right]$. In this simple example we also calculate $\tilde{k}_{t}$ explicitly, although the computational effort required suggests that in more complex situations it may not be a good idea to do so.

First, we see how stochastic integrals with respect to $M$ look like. Recall that, by proposition $1.9 \tilde{A}_{t}=a(t \wedge \tau)$, where $a: \mathbb{R}^{+} \rightarrow \mathbb{R}^{+}$.

Lemma 5.2. Let $k_{t}$ be a $\mathcal{F}^{\tau}$-measurable process. Then we have:

$$
\int_{0}^{T} k_{t} d M_{t}=k_{\tau}(\tau) 1_{\{\tau \leq T\}}-\int_{0}^{T \wedge \tau} k_{t}(t) d a_{t}
$$

Proof. By Corollary 1.3, we have that any $\mathcal{F}^{\tau}$-measurable process can be written as $k_{t}(t \wedge \tau)$. Hence:

$$
\begin{aligned}
\int_{0}^{T} k_{t}(t \wedge \tau) d M_{t} & =k_{\tau}(\tau) 1_{\{\tau \leq T\}}-\int_{0}^{T} k_{t}(t \wedge \tau) d a_{t \wedge \tau}= \\
& =k_{\tau}(\tau) 1_{\{\tau \leq T\}}-\int_{0}^{T \wedge \tau} k_{t}(t \wedge \tau) d a_{t}= \\
& =k_{\tau}(\tau) 1_{\{\tau \leq T\}}-\int_{0}^{T \wedge \tau} k_{t}(t) d a_{t}
\end{aligned}
$$


The above lemma shows that $k_{t}(s)$ needs only be defined for $s=t$, so from now on we shall unambiguously write $k_{t}$ instead of $k_{t}(t)$.

Now we can compute $\tilde{k}_{t}$ :

Proposition 5.3. $\tilde{k}_{t}$ is the unique solution of the following ODE:

$$
\left\{\begin{array}{l}
k_{t}^{\prime}=\alpha+\left(a_{t}^{\prime}+\alpha\right) k_{t}+a_{t}^{\prime} k_{t}^{2} \\
k_{0}=\frac{\exp \left(-\lambda_{2}^{2} T\right)}{c}-1
\end{array}\right.
$$

where

$$
c=\exp \left(-\lambda_{2}^{2} T\right) \int_{0}^{T} \exp (\alpha t) d F_{t}+\exp \left(-\lambda_{1}^{2} T\right)\left(1-F_{T}\right)
$$

and $F_{t}=P(\tau \leq t)$.

Proof. By lemma 5.2, and the generalization of Itô's formula for processes with jumps, we have:

$$
\begin{aligned}
\mathcal{E}\left(\int_{0}^{T} k_{t}(\eta) d M_{t}\right)=\mathcal{E}\left(k_{\tau} 1_{\{\tau \leq T\}}-\int_{0}^{T \wedge \tau} k_{t} d a_{t}\right)= \\
=\left(1+k_{\tau} 1_{\{\tau \leq T\}}\right) \exp \left(-\int_{0}^{\tau \wedge T} k_{t} d a_{t}\right)
\end{aligned}
$$

Hence, by 3.2, we have:

$$
\left(1+k_{\tau} 1_{\{\tau \leq T\}}\right) \exp \left(-\int_{0}^{\tau \wedge T} k_{t} d a_{t}\right)=\frac{\exp \left(-T \lambda_{1}^{2}-(T-\tau \wedge T) \alpha\right)}{E\left[\exp \left(-\int_{0}^{T} \lambda_{t}^{2} d t\right)\right]}
$$

Taking logarithms of both sides, and setting $c=E\left[\exp \left(-\int_{0}^{T} \lambda_{t}^{2} d t\right)\right]$, we get:

$$
\ln \left(1+k_{\tau} 1_{\{\tau \leq T\}}\right)-\int_{0}^{\tau \wedge T} k_{t} d a_{t}=-T \lambda_{1}^{2}-(T-\tau \wedge T) \alpha-\ln c
$$

Differentiating with respect to $\tau$, for $\tau \leq T$ we obtain equation (7).

Remark 5.4. Equation (7) is a Riccati ODE, and can be solved in terms of the function $a_{t}$. Depending on the form of $a_{t}$, explicit solutions may or may not be available.

5.2.2. $\alpha$ Bernoulli. In this case, $\alpha$ is a Bernoulli random variable, independent of $\tau$, with values $\left\{\alpha_{0}, \alpha_{1}\right\}$. We also set $A=\left\{\alpha=\alpha_{0}\right\}$, $B=\left\{\alpha=\alpha_{1}\right\}$, and $p=P(B)$. Since the support of $\alpha$ is no longer a single point, a martingale will not be sufficient for representation purposes. In fact, two martingales do the job, as we prove in the following:

Proposition 5.5. Let $N_{t}=1_{\{\tau \leq t\}}\left(1_{B}-p\right)$. Then the set of two martingales $\{M, N\}$ has the representation property. 
Proof. First we check that $M$ and $N$ are orthogonal. This is easily seen, since $M N=N a_{\tau}$. We now prove that the martingale measure is unique.

Let $Q$ be a martingale measure for $\{M, N\}$. As shown in the proof of Proposition 5.1, the distribution of $\tau$ under $Q$ must be the same as under $P$. However, we also need that $Q(B)=P(B)$, otherwise $N$ would not be a martingale.

The change from $P$ to $\tilde{P}$ is a change in the joint law of $(\tau, \alpha)$. Under $P$ this is a product measure, since $\tau$ and $\alpha$ are independent. However, we cannot expect that the same holds under $\tilde{P}$. For $t \leq T$ we have:

$$
\frac{d \tilde{P}_{H}}{d P_{H}}=\frac{1_{A} \exp \left(-\lambda_{1}^{2} T-(T-t) \alpha_{0}\right)+1_{B} \exp \left(-\lambda_{1}^{2} T-(T-t) \alpha_{1}\right)}{c}
$$

Therefore the law of $\tau$ under $\tilde{P}$ is given by:

$\tilde{f}_{t}=\frac{1}{c}\left(p \exp \left(-\lambda_{1}^{2} T-(T-t) \alpha_{1}\right)+(1-p) \exp \left(-\lambda_{1}^{2} T-(T-t) \alpha_{0}\right)\right) f_{t}$

And the conditional law of $\alpha$ with respect to $\tau$ is given by:

$$
\begin{aligned}
& \tilde{P}(B \mid \tau \in d t)= \\
& \quad=\frac{p \exp \left(-\lambda_{1}^{2} T-(T-t) \alpha_{1}\right)}{\left(p \exp \left(-\lambda_{1}^{2} T-(T-t) \alpha_{1}\right)+(1-p) \exp \left(-\lambda_{1}^{2} T-(T-t) \alpha_{0}\right)\right)}
\end{aligned}
$$

In particular, it is immediately seen that $\alpha$ is independent of $\tau$ if and only if it degenerates in the previous case.

When $\alpha$ is Bernoullian, calculating $k$ involves solving a system of two Riccati ODEs, which is somewhat cumbersome. More generally, if the support of $\alpha$ is made of $n$ points, it is reasonable that $n$ martingales are required for representation purposes. As a result, the values of $k$ would be the solutions of a system of $n$ ODEs.

5.2.3. $\alpha$ continuously distributed. In this case the support of $\alpha$ is an infinite set, therefore Theorem 3.1 is no longer applicable. In fact we need its random measure analogous, given by Theorem 4.1. If the filtration $\mathcal{F}^{\lambda}$ generated by $\lambda_{t}$ coincides with the one generated by $\mu_{t}$ and $\sigma_{t}$, we can assume $\mathcal{F}^{H}=\mathcal{F}^{\lambda}$. By Proposition 1.17, there exists a random measure $\nu$ associated to $\lambda$, and given by:

$$
\nu(\eta ; d t, d x)=\epsilon_{\{\tau, \alpha(\eta)\}}(d t, d x)
$$

Since this is a multivariate point process, and $\mathcal{F}^{H}$ coincides with the smallest filtration under which $\nu$ is optional, by Theorem 1.22 the compensated measure $\nu-\nu^{p}$ has the representation property on $H$. Also, $k *\left(\nu-\nu^{p}\right)$ is a purely discontinuous martingale for all $\tilde{\mathcal{P}}$-measurable processes $k$, therefore $\left[W, k *\left(\nu-\nu^{p}\right)\right]=0$. This means that the assumptions of Theorem 4.1 are satisfied, and $\tilde{P}$ is given by Proposition 4.4 . 
Suppose that $\alpha$ has a density, say $g(x)$. We have:

$$
\frac{d \tilde{P}_{H}}{d P_{H}}=h(t, x)=\frac{\exp \left(-\lambda_{1}^{2} T-x(T-t)\right)}{c}
$$

Denoting by $j(t, x)$ and $\tilde{j}(t, x)$ the joint densities of $(\tau, \alpha)$ under $P$ and $\tilde{P}$ respectively, we have:

$$
\begin{aligned}
\tilde{j}(t, x) & =h(t, x) j(t, x)=h(t, x) f(t) g(x) \\
\tilde{f}(t) & =f(t) \int h(t, x) g(x) d x \\
\tilde{g}(x \mid t) & =\frac{j(t, x)}{\tilde{f}(t)}=\frac{h(t, x) g(x)}{\int h(t, x) g(x) d x}
\end{aligned}
$$

If $\alpha \sim \mathcal{N}(\delta, v)$, the density of $\tau$ under $\tilde{P}$ is given by:

$$
\begin{aligned}
\tilde{f}_{t}=f_{t} \int_{-\infty}^{+\infty} \frac{1}{c} \exp \left(-\lambda_{1}^{2} T-\alpha(T-t)\right) n\left(\frac{\alpha-\delta}{\sqrt{v}}\right) d \alpha= \\
=\frac{1}{c} \exp \left(-\lambda_{1}^{2} T-\delta(T-t)+\frac{1}{2}(T-t)^{2} v\right) f_{t}
\end{aligned}
$$

where $n(x)$ is the standard normal density function. It is easy to check that the conditional density of $\alpha$ given $\tau$ is of the form:

$$
\tilde{g}(x \mid t)=\frac{1}{\sqrt{2 \pi v}} \exp \left(-\frac{1}{2} \frac{(x-(\alpha+(T-t) v))^{2}}{v}\right)
$$

Therefore $\alpha$ is conditionally normal under $\tilde{P}$, with distribution $\mathcal{N}(\delta+(T-t) v, v)$.

Remark 5.6. In the specific case of $\lambda_{t}$ being normally distributed, it can be shown that $G_{T}(\Theta)$ is not closed, therefore Proposition 3.2 does not hold. However, in [1] the above formula is proved relaxing this assumption. It is also shown that in this example $G_{T}(\Theta)$ is closed if and only if the support of $\alpha$ is bounded from above.

5.2.4. Multiple random jumps. Leaving $\alpha$ deterministic for simplicity, we now study the following model:

$$
\lambda_{t}^{2}=\lambda_{0}^{2}+\sum_{i=1}^{n} 1_{\left\{t \geq \tau_{i}\right\}} \alpha_{i}
$$

We assume that $\tau_{i+1}-\tau_{i}$ are IID random variables, with common density $f(x)$. Denote by $H$ the space $[0, T]^{n}$, endowed with the image measure of the mapping $\left(\tau_{1}, \ldots, \tau_{n}\right) \mapsto\left(\tau_{1} \wedge T, \ldots, \tau_{n} \wedge T\right)$, and with the natural filtration $\mathcal{F}_{t}$ generated by $\left\{\tau_{1} \wedge t, \ldots, \tau_{n} \wedge t\right\}$. A martingale with the predictable representation property is given by $M_{t}=\sum_{i=1}^{n} 1_{\left\{t \geq \tau_{i}\right\}}$. In this case, the density of $\tilde{P}$ is given by:

$$
\frac{d \tilde{P}_{H}}{d P_{H}}=\frac{\exp \left(-\lambda_{0}^{2} T-\sum_{i=1}^{n} \alpha_{i}\left(T-\tau_{i}\right) \wedge 0\right)}{c}
$$


Since this density cannot be factored into a product of densities each one involving at most a $\tau_{i}-\tau_{i-1}$, it follows that under $\tilde{P}$ the increments of the stopping times are no longer independent.

For example, consider the following case, with $n=2$ and the stopping times exponentially distributed with parameter $b$. In other words:

$$
\left\{\begin{array}{l}
P(x \in d t)=b e^{-b t} \quad \text { for } t<T \\
P(x=T)=e^{-b T}
\end{array}\right.
$$

for $x=\tau_{1}, \tau_{2}-\tau_{1}$. We obtain that:

$$
\left\{\begin{array}{l}
\tilde{P}\left(\tau_{1} \in d t\right)=\frac{\exp \left(-\left(\lambda_{0}^{2}+\alpha\right) T\right)}{c} e^{-(b-\alpha) t} \\
\tilde{P}\left(\tau_{1}=T\right)=\frac{\exp \left(-\left(\lambda_{0}^{2}+b\right) T\right)}{c}
\end{array}\right.
$$

The conditional law of $\tau_{2}-\tau_{1}$ turns out to be of the same form of (8) where $T$ is replaced by $T-\tau_{1}$. This shows that under $\tilde{P}$ the law of $\tau_{2}$ is not independent of $\tau_{1}$.

\section{The optimal STRATEGy FOR A CALL OPtion}

An elegant approach to the construction of the optimal strategy of a call option was presented in El Karoui-Geman-Rochet [8], where the technique of change of numeraire is introduced. We now adapt this method to write explicitly the optimal strategy for a call option.

Throughout this section, we shall make the following assumptions:

i) The space $G_{T}(\Theta)$ is closed in $L^{2}(P)$.

ii) $\mu_{t}$ and $\sigma_{t}$ depend only on $\eta$ (in particular, $(\beta)$ holds).

Condition $i$ ) guarantees the existence of the optimal strategy for any option $H$ such that $\frac{H}{B_{T}} \in L^{2}(P)$, as shown

$$
\frac{d \tilde{P}}{d P}=\mathcal{E}\left(-\int_{0} \lambda_{s} d W_{s}\right)_{T} \frac{\exp \left(-\int_{0}^{T} \lambda_{t}^{2} d t\right)}{E\left[\exp \left(-\int_{0}^{T} \lambda_{t}^{2} d t\right)\right]}
$$

Recall that under the filtration $\tilde{\mathcal{F}}_{t}=\mathcal{F}_{t} \otimes \mathcal{E}$ the model is complete: for instance, a "call" option of strike $K$ on the

$$
X_{t} N\left(d_{1}\left(t, \eta, X_{t}\right)\right)-\frac{K}{B_{T}} N\left(d_{2}\left(t, \eta, X_{t}\right)\right)
$$

where $N(\cdot)$ is the distribution function of the standard normal variable and

$$
d_{1,2}(t, \eta, x)=\frac{\ln \left(\frac{x}{K B(t, T)}\right) \pm \int_{t}^{T} \sigma^{2}(s, \eta) d s}{\left(\int_{t}^{T} \sigma^{2}(s, \eta) d s\right)^{\frac{1}{2}}}
$$

with $B(t, T)=\frac{B_{t}}{B_{T}}$. We observe that for all $t$, the filtration $\tilde{\mathcal{F}}_{t}$ contains the information on volatility up to $T$ : more precisely, the random 
variable $\int_{t}^{T} \sigma^{2}(s, \eta) d s$ is $\tilde{\mathcal{F}}_{t}$-measurable. It is easy to see that the probability $\tilde{P}$ is an equivalent martingale measure with respect to $\tilde{\mathcal{F}}_{t}$ : in fact, with the same argument as El Karoui-Geman-Rochet [8], we obtain that (recalling that $X_{t}$ denotes the discounted price of $S_{t}$ ):

$$
\tilde{E}\left[\left(X_{T}-\frac{K}{B_{T}}\right)^{+} \mid \tilde{\mathcal{F}}_{t}\right]=X_{t} \tilde{E}^{X}\left[1_{A} \mid \tilde{\mathcal{F}}_{t}\right]-\frac{K}{B_{T}} \tilde{E}\left[1_{A} \mid \tilde{\mathcal{F}}_{t}\right]
$$

where $A=\left\{S_{T}>K\right\}$ and $\tilde{E}^{X}$ denotes the expectation under the probability $\tilde{P}^{X}$, defined as:

$$
\frac{d \tilde{P}^{X}}{d \tilde{P}}=\frac{X_{T}}{E\left[X_{T}\right]}
$$

which, as observed by Gourieroux, Laurent and Pham, is the varianceoptimal martingale measure for the numeraire $X_{t}$. In particular, the only replicating portfolio is given by:

$$
\tilde{\xi}_{t}^{1} X_{t}-\frac{K}{B_{T}} \tilde{\xi}_{t}^{2}
$$

where $\tilde{\xi}_{t}^{1}=\tilde{E}^{X}\left[1_{A} \mid \tilde{\mathcal{F}}_{t}\right]$ and $\tilde{\xi}_{t}^{2}=\tilde{E}\left[1_{A} \mid \tilde{\mathcal{F}}_{t}\right]$. Let $\xi_{t}^{1}$ and $\xi_{t}^{2}$ the predictable projections of $\tilde{\xi}_{t}^{1}$ and $\tilde{\xi}_{t}^{2}$ with respect to the filtration $\tilde{\mathcal{F}}_{t}$ and the probability $\tilde{P}$. By the same argument used in Biagini and Pratelli ([2], Proposition 5.1), it follows that $\xi_{t}^{1}$ coincides with the predictable projection of the process $Y(t, \omega)=1_{A}(\omega)$ with respect to the probability $\tilde{P}^{X}$ and the filtration $\mathcal{F}_{t}$. More precisely, for all predictable stopping times $\tau$ we have:

$$
\xi_{\tau}^{1}=\tilde{E}^{X}\left[1_{A} \mid \mathcal{F}_{\tau-}\right]
$$

Note that the left-continuous versions of the stochastic processes $\tilde{E}^{X}\left[1_{A} \mid \mathcal{F}_{t-}\right]$ and $\tilde{E}\left[1_{A} \mid \mathcal{F}_{t-}\right]$ always exists and they coincide with the predictable projections $\xi_{t}^{1}$ and $\xi_{t}^{2}$. We are now ready to state the following

Proposition 6.1. If $S_{T}$ is square-integrable with respect to $\tilde{P}$, the optimal strategy $\theta_{t}$ is given in the following feedback form:

$$
\theta_{t}=\xi_{t}^{1}-\frac{\lambda_{t}}{\sigma_{t} X_{t}}\left(\xi_{t}^{1} X_{t}-\frac{K}{B_{T}} \xi_{t}^{2}-\tilde{E}\left[\frac{\left(S_{T}-K\right)^{+}}{B_{T}}\right]-\int_{0}^{t} \theta_{s} d X_{s}\right)
$$

Proof. The formula for $\theta$ follows from Theorem 6 in Rheinländer and Schweizer [16]. The expression for $\theta$ is:

$$
\theta_{t}=\tilde{\xi}_{t}-\frac{\tilde{\zeta}_{t}}{\tilde{Z}_{t}}\left(\tilde{V}_{t-}-\int_{0}^{t} \theta_{s} d X_{s}\right)
$$


From the equality:

$$
\frac{d \tilde{P}}{d P}=\frac{\mathcal{E}\left(-\int_{0}^{T} \frac{\lambda_{s}}{\sigma_{s} X_{s}} d X_{s}\right)}{E\left[\mathcal{E}\left(-\int_{0}^{T} \frac{\lambda_{s}}{\sigma_{s} X_{s}} d X_{s}\right)\right]}
$$

we obtain that $\frac{\tilde{\zeta}_{t}}{\tilde{Z}_{t}}=\frac{\lambda_{s}}{\sigma_{s} X_{s}}$. Also,

$$
\tilde{V}_{t}=\tilde{E}\left[\frac{\left(S_{T}-K\right)^{+}}{B_{T}} \mid \mathcal{F}_{t}\right]=X_{t} \tilde{E}^{X}\left[1_{A} \mid \mathcal{F}_{t}\right]-\frac{K}{B_{T}} \tilde{E}\left[1_{A} \mid \mathcal{F}_{t}\right]
$$

By assumption, $\tilde{V}_{t^{-}}=X_{t} \xi_{t}^{1}-\frac{K}{B_{T}} \xi_{t}^{2}$. Finally,

$$
\frac{H}{B_{T}}=\frac{\left(S_{T}-K\right)^{+}}{B_{T}}=\tilde{E}\left[\frac{H}{B_{T}} \mid \tilde{\mathcal{F}}_{0}\right]+\int_{0}^{T} \tilde{\xi}_{s}^{1} d X_{s}
$$

Then, from Theorem 2.5 in Schweizer (1993), we obtain:

$$
\frac{H}{B_{T}}=E\left[\frac{H}{B_{T}}\right]+\int_{0}^{T} g_{t} d X_{s}+L_{t}^{H}
$$

where $g_{t}$ is the $\mathcal{F}_{t}$-predictable projection of $\tilde{\xi}_{t}^{1}$ (calculated under $\tilde{P}$ ) and $L_{t}^{H}$ is a $\tilde{P}$-However, $\tilde{\xi}_{t}^{1}$ is the optional projection (with respect to $\tilde{P}^{X}$ and the filtration $\tilde{\mathcal{F}}_{t}$ ) of the process

Remark 6.2. The application of Proposition 6.1 in concrete cases involves the calculation of the terms:

$$
\begin{aligned}
\tilde{E}^{X}\left[1_{A} \mid \mathcal{F}_{t-}\right] & =\tilde{E}\left[\tilde{E}^{X}\left[1_{A} \mid \mathcal{F}_{t}\right] \mid \tilde{\mathcal{F}}_{t-}\right] \\
\tilde{E}\left[1_{A} \mid \mathcal{F}_{t-}\right] & =\tilde{E}\left[\tilde{E}\left[1_{A} \mid \mathcal{F}_{t}\right] \mid \tilde{\mathcal{F}}_{t-}\right]
\end{aligned}
$$

we know that:

$$
\begin{aligned}
\tilde{E}^{X}\left[1_{A} \mid \mathcal{F}_{t}\right] & =N\left(d_{1}\left(t, \eta, X_{t}\right)\right) \\
\tilde{E}\left[1_{A} \mid \mathcal{F}_{t}\right] & =N\left(d_{2}\left(t, \eta, X_{t}\right)\right)
\end{aligned}
$$

Denoting by $P_{E}$ and $\tilde{P}_{E}$ respectively the projections of $P$ and $\tilde{P}$ on $E$, we have:

$$
\frac{d \tilde{P}_{E}}{d P_{E}}=\frac{\exp \left(-\int_{0}^{T} \lambda_{t}^{2} d t\right)}{E\left[\exp \left(-\int_{0}^{T} \lambda_{t}^{2} d t\right)\right]}
$$

Recalling that $X_{t}$ is $\mathcal{F}_{t^{-}}$-measurable, we obtain, for $i \in\{1,2\}$ :

$$
\tilde{E}\left[N\left(d_{i}\left(t, \eta, X_{t}\right)\right) \mid \mathcal{F}_{t-}\right]=F_{i}\left(t, \eta, X_{t}\right)
$$

here:

$$
F_{i}(t, \eta, x)=\tilde{E}\left[N\left(d_{i}(t, \eta, x)\right) \mid \mathcal{F}_{t-}\right]=\tilde{E}\left[N\left(d_{i}(t, \eta, x)\right) \mid \mathcal{E}_{t-}\right]
$$


For instance, consider example 5.1 with $n=1$. In this case, $\eta$ is a Bernoulli random variable under $\tilde{P}$, and we denote $\tilde{p}=\tilde{P}(\eta=0)$. The strategy is given by:

$$
\xi_{t}^{1}=\left(\tilde{p} \xi_{t}^{1}(0)+(1-\tilde{p}) \xi_{t}^{1}(1)\right) 1_{\left\{t<t_{1}\right\}}+\xi_{t}^{1}(\eta) 1_{\left\{t \geq t_{1}\right\}}
$$

In a similar fashion, the optimal strategy can be calculated in more complex examples, the computational effort becoming correspondingly higher.

\section{ACKnowledgements}

We wish to thank Maurizio Pratelli for proposing this research, and for his precious advice in many discussions. We also thank Paul Embrechts, for suggesting some examples, and Koichiro Takaoka for his comments, and for a careful reading of an earlier version of the paper.

\section{REFERENCES}

[1] F. Biagini, P. Guasoni, and M. Pratelli. Mean-variance hedging for stochastic volatility models. Preprint, April 1999.

[2] F. Biagini and M. Pratelli. Local risk minimization and numèraire. Journal of Applied Probability, 1999. To appear.

[3] F. Delbaen and W. Schachermayer. The variance-optimal martingal measure for continuous precesses. Bernoulli, pages 81-105, 1996.

[4] C. Dellacherie and P.A. Meyer. Probabilities and Potential. North-Holland, Amsterdam, 1978.

[5] C. Dellacherie and P.A. Meyer. Probabilities and Potential B: Theory of Martingales. North-Holland, Amsterdam, 1982.

[6] D. Duffie and H. R. Richardson. Mean-variance hedging in continuous time. Annals of Applied Probability, 1:1-15, 1991.

[7] H. Föllmer and D. Sondermann. Hedging of non-redundant contingent claims. In W. Hildenbrand and A. Mas-Colell, editors, Contribution to Mathematical Economics, pages 205-223. 1986.

[8] H. Geman, N. El Karoui, and J.C. Rochet. Changes of numéraire, changes of probability measure and ion pricing. J. Appl. Prob., 32:443-458, 1995.

[9] S. Heston. A closed-form solution for option with stochastic volatility with applications to bond and currency options. Review of Financial Studies, 6:327343, 1993.

[10] C. Hipp. Hedging general claims. In Proceeding of the $3^{\text {rd }}$ AFIR Colloquium, volume 2, pages 603-613, Rome, 1993.

[11] J. Hull and A. White. The pricing of options on assets with stochastic volatilities. Journal of Finance, 42:281-300, 1987.

[12] J.Jacod and A.N.Shiryaev. Limit Theorems for Stochastic Processes. SpringerVerlag, 1987.

[13] P. Monat and C. Stricker. Föllmer-schweizer decomposition and mean-variance hedging for general claims. annals of Probability, 23:605-628, 1995.

[14] H. Pham, T. Rheinländer, and M. Schweizer. Mean-variance hedging for continuous processes: New proofs and examples. Finance and Stochastics, 2:173198, 1997.

[15] P. Protter. Stochastic Integration and Differential Equations: A New Approach. Springer-Verlag, 1990. 
[16] T. Rheinländer and M. Schweizer. On $l^{2}$-projections on a space of stochastic integrals. Annals of Probability, 25(4):1810-1831, 1997.

[17] M. Schäl. On quadratic cost criteria for option hedging. Mathematics of Operations Research, 19:121-131, 1994.

[18] M. Schweizer. Option hedging for semimartingales. Stochastic Processes and their Appl., 37:339-363, 1991.

[19] M. Schweizer. Mean-variance hedging for general claims. Annals of Applied Probability, 2:171-179, 1992.

[20] M. Schweizer. Risk-minimizing hedging strategies under restricted information. Math. Finance, 4:327-342, 1994.

[21] M. Schweizer. Approximation pricing and the variance-optimal martingale measure. Annals of Probability, 64:206-236, 1996.

[22] E. M. Stein and J. C. Stein. Stock price distributions with stochastic volatility: an analytic approach. Review of Financial Studies, 4:727-752, 1991.

[23] Peter Zangari. An improved methodology for measuring var. RiskMetrics Monitor, 2:7-25, 1996.

Scuola Normale Superiore P.zza Dei Cavalieri, 756126 Pisa Italy

E-mail address: guasoni@sns.it

Dipartimento di Matematica Piazza Porta S. Donato 40127 Bologna ITALY

E-mail address: biagini@dm.unibo.it 